\title{
Manutenção da prática de exercícios de reeducação de membros superiores por estudantes de Fisioterapia acompanhamento de seis meses
}

\section{Extremity superior exercises practice among Physiotherapy students - six-month follow-up study}

\author{
Fátima Aparecida Caromano1, Marina Carlis², Liedi dos Santos \\ Cordeiro Panades ${ }^{3}$, Maiza Ritomi Ide ${ }^{4}$, \\ Silvana Maria Blascovi de Assis ${ }^{5}$
}

\begin{abstract}
CAROMANO, F. A.; CARLIS, M.; PANADES, L. dos S. C.; IDE, M. R.; ASSIS, S. M. B. de. Manutenção da prática de exercícios de reeducação de membros superiores por estudantes de Fisioterapia - acompanhamento de seis meses. Rev. Ter. Ocup. Univ. São Paulo, v.19, n. 1, p. 56-60, jan./abr. 2008.
\end{abstract}

RESUMO: Objetivo: Avaliar a manutenção e os efeitos da prática de exercícios de reeducação de membros superiores, por um período de seis meses, por estudantes de Fisioterapia. Local: Este estudo foi conduzido de 1999 a 2003, com cinco grupos de 10 estudantes de Fisioterapia - Curso de Fisioterapia, Faculdade de Medicina, Universidade de São Paulo. Participantes: 50 estudantes, 10 homens e 40 mulheres (idade média de 20,4 2,1 anos) matriculados na disciplina de Recursos Terapêuticos Manuais. Procedimento: O programa consistiu em uma seqüência de 20 exercícios que tiveram por objetivo o ganho de força muscular, treinados em padrões funcionais de movimentos. Os exercícios foram treinados durante dez sessões de uma hora que consistiu em 20 minutos para discussão de estudos que mostravam lesões musculoesqueléticas em profissionais fisioterapeutas e 40 minutos de exercícios. Avaliação: Oito testes de força muscular isométrica de flexão e extensão de membro superior direito foram realizados por meio de dinamometria digital (Lafayetti Instruments). Uma questão aberta e o pedido para demonstração de seqüência de exercícios, como treinado, foram usados para avaliar o comportamento. Análise de dados: $\mathrm{O}$ estudo do efeito do programa usou a média dos oito testes de força muscular do membro superior direito obtida em T1 (antes do treinamento) e comparou com a obtida em T2 (após o treinamento) usando o Teste $t$ de

\footnotetext{
1. Profa Dra do Curso de Fisioterapia da FMUSP.

2. Graduanda em Fisioterapia.

3. Graduanda em Fisioterapia.

4. Profa. Ms do Curso de Fisioterapia da Universidade do Oeste do Paraná.

5. Profa. do Programa de Pós-Graduação em Desenvolvimento Humano e Coordenadora do Curso de Graduação em Fisioterapia da Universidade Presbiteriana Mackenzie.

Endereço para correspondência: Depto de Fisioterapia, Fonoaudiologia e Terapia Ocupacional da FMUSP Curso de Fisioterapia LaFi. Com, A/C Fátima Caromano. Rua Cipotânea, 51. São Paulo, SP, Cidade Universitária da USP. CEP: 05360-000.
} 
Student pareado. O mesmo teste foi utilizado para comparar T2 e T3, resultando em um indicativo de manutenção. As diferenças encontradas foram transformadas em porcentagem. Foi realizada a comparação entre os subgrupos de homens e mulheres. As respostas para a pergunta aberta foram estudadas quantitativamente e as respostas foram agrupadas por assunto. Resultados: Quarenta e oito dos 50 estudantes que iniciaram o programa participaram da avaliação seis meses após o término do programa. O programa produziu uma melhora estatisticamente significante em força muscular $(\mathrm{p}=0,0067)$ com aumento de $32 \%$. Trinta e dois participantes informaram ter continuado a praticar de alguma forma e outros 17 relataram não ter praticado os exercícios treinados durante os seis meses precedentes. Razões para manutenção envolveram intenção de prevenção e melhor controle de movimentos dos membros superiores. Razões para abandono incluíram falta de tempo e outras prioridades. O gênero não afetou nem os resultados relativos à força muscular, nem o comportamento de manutenção. Conclusões: O programa proposto produziu melhora significativa na força muscular. A manutenção se deu em índices superiores aos encontrados na literatura. Os comportamentos envolvidos com manutenção se relacionaram com as discussões teóricas propostas no programa. A forma de ensino e treinamento foi eficiente para memorização, produzindo alto índice de reprodutibilidade correta destes seis meses após o final do programa. A manutenção da prática de exercícios implicou em melhora adicional. Acreditamos que este tipo de atividade deva ser oferecido como rotina para alunos de cursos de Fisioterapia.

DESCRITORES: Fisioterapia. Exercício. Estudantes de ciências da saúde. Manutenção preventiva. Terapia por exercício/recursos humanos. Membro superior. Força muscular.

\section{INTRODUÇ̃̃O}

$\mathrm{A}$ derência a um programa de exercício físico tem sido definido como uma prática compor-

tamental dentro de um programa estruturado, enquanto que, manutenção seria a continuidade da prática dos exercícios fora do programa formal ${ }^{1,2}$.

De forma geral, a manutenção da prática de exercícios físicos referida na literatura é inferior a $50 \%$, sendo que os valores maiores são encontrados em programas que conseguem promover uma mudança claramente observada pelo participante, a exemplo das quedas no nível sérico de colesterol, na percepção da dor ou no peso corporal. Programas preventivos, sem agravantes históricos ou familiares, têm baixa porcentagem de participantes que fazem a manutenção de forma adequada $a^{3,4,5}$.

A manutenção da qualidade do desempenho físico é uma questão de sobrevivência para o profissional fisioterapeuta. Durante a graduação, o fisioterapeuta desenvolve habilidades de autocuidado postural, mas nem sempre tem força muscular adequada para executar técnicas como mudança de decúbito ou massoterapia. $\mathrm{O}$ alerta se refere às questões ligadas à prevenção, pois as atividades realizadas pelos fisioterapeutas requerem esforços constantes e repetitivos que podem produzir lesões ou levar à exaustão, como já foi demonstrado em diferentes estudos ${ }^{6,7,8,9}$.

$\mathrm{O}$ ensino e o treinamento de habilidades profissionalizantes é uma preocupação constante na formação destes profissionais, no entanto, não existem estudos avaliando a manutenção de práticas físicas preventivas.

Com base no exposto, o objetivo deste estudo foi avaliar a manutenção e os efeitos de um programa supervisionado de exercícios de fortalecimento de membros superiores para estudantes de Fisioterapia, seis meses após o final dos treinamentos.

\section{MÉTODO}

Participantes: $\mathrm{O}$ estudo foi conduzido com a participação voluntária de 50 estudantes, regularmente matriculados na disciplina de Recursos Terapêuticos Manuais, 10 homens e 40 mulheres (idade média de 20,4 $\pm 2,1$ anos, peso médio de 70,2 $\pm 3,6 \mathrm{Kg}$, altura média 1,63 $\pm 0,4 \mathrm{~m}$ ).

Foram critérios de inclusão no estudo o interesse declarado de participar do programa, queixa de cansaço dos membros superiores durante a prática de massoterapia, ser destro, relatar não ter queixas de ordem musculoesquelética e apresentar amplitude de movimento de membros superiores, mensurada por goniometria, dentro de parâmetros da normalidade.

Local: Este estudo foi conduzido entre os anos 1999 e 2003, no Laboratório de Fisioterapia e Comportamento (LaFi.Com) do Curso de Fisioterapia da Faculdade de Medicina da Universidade de São Paulo. 
Intervenção: O programa constou de uma seqüência de 20 exercícios de que tinham por objetivo o ganho de força muscular, treinados em padrões funcionais de movimentos. Os exercícios foram treinados em dez sessões de uma hora que constavam de 20 minutos para discussão de artigos mostrando lesões ocupacionais musculoesqueléticas em profissionais fisioterapeutas visando mostrar a alta incidência destas e motivação para treinamento e manutenção, seguidos de 40 minutos para treinamento de exercícios.

Avaliação: $\mathrm{O}$ estudo foi delineado para determinar a força muscular do membro superior dominante e os fatores envolvidos com a manutenção da prática de exercícios em follow-up de seis meses.

A testagem da força muscular isométrica de oito grupos musculares (flexores e extensores) do membro superior direito, mensurados por meio de dinamometria digital ou miometria (Lafayette Instruments) foi usada como indicador da manutenção de exercícios, bem como para avaliação do efeito do treinamento ${ }^{10,11}$.

Foram coletadas três medidas de cada teste, sendo utilizada, para fins de estudo, a maior medida obtida. As oito medidas coletadas foram somadas e divididas por oito, obtendo se uma média da força muscular no membro superior direito.

Procedimentos: Foram selecionados e treinados cinco grupos com 10 participantes cada. Após conhecimento e aceite de participação na pesquisa os participantes se submeteram a avaliação de força muscular de oito grupos musculares de membro superior direito $\left(1^{\circ}\right.$ avaliação- T1) e em seguida iniciaram o treinamento supervisionado. Uma semana $\left(2^{\circ}\right.$ avaliação- T2) e seis meses após o final do treinamento ( $3^{\circ}$ avaliação - T3) os participantes foram reavaliados. Em T3, foi aplicada uma questão aberta sobre a manutenção e fatores de manutenção das práticas de exercícios para membros superiores no período estudado e foi requisitada a replicação da seqüência de exercícios treinados.

Análise de dados: Para estudo do efeito do programa a média da força muscular do membro superior direito obtida em T1 foi comparada com as obtidas em T2, utilizando-se o Teste $t$ de Student pareado. A diferença encontrada também foi transformada em porcentagem. O mesmo procedimento foi utilizado para gerar indicadores de manutenção, e comparou-se então, os tempos T2 e T3. Também foi comparado o desempenho dos subgrupos masculino e feminino. A questão aplicada foi estudada quantitativamente sendo que a informações coletadas foram agrupadas por assunto. O estudo tratou os dados obtidos pelas respostas na questão aberta de forma quantitativa, estudo clínico $\square$ qualitativo/ quantitativo (TURATO, 2003) oferecendo um panorama geral das informações gerada em porcentagem.

O conteúdo das respostas á questão apresentada foi analisado primeiramente de forma qualitativa, permitindo a criação de categorias de respostas relevantes ao estudo, (neste caso, abandono ou manutenção), conforme premissa de Bogdan e Biklen (4). Estes autores também colocam a importância de descrição de situações e acontecimentos envolvidos no estudo, o que foi contemplado com requisição de replicação dos exercícios treinados.

\section{RESULTADOS E DISCUSSÃO}

Quarenta e oito dos cinqüenta sujeitos que participaram do estudo completaram a avaliação realizada seis meses após o final do treinamento. Dois alunos mudaram de curso de graduação e não mostraram interesse em participar da avaliação final. $\mathrm{O}$ treinamento produziu melhora estatisticamente significativa na força muscular do membro superior direito $(p=0,0067)$, usado como referência de melhora em membros superiores pelo fato dos participantes serem destros, com aumento médio de força para o grupo de $32 \%$. Estes dados demonstram que o programa proposto foi eficiente em produzir ganhos de força muscular, atingindo o objetivo a que se propôs.

Trinta e um dos quarenta e oito participantes (65\%) relataram que deram continuidade, de alguma forma, à prática de exercícios de membros superiores. Destes, onze treinaram os exercícios de acordo com o proposto pelo programa, pelo menos duas vezes por semana, enquanto que 20 referiram que associaram o treinamento com uma prática esportiva ou atividade em academia, pelo menos uma vez por semana. Dezessete estudantes (35\%) disseram que não deram continuidade a pratica de exercícios de membros superiores. A porcentagem de sujeitos que mantiveram o programa foi maior do que o esperado, considerando-se as expectativas geradas pela literatura, que é de menos de $50 \%$ de manutenção. Associamos este achado à inserção de discussão de textos que abordava diferentes tipos de lesões ocupacionais apresentadas por fisioterapeutas (12), o que foi confirmado nos relatos escritos referentes às razões de manutenção.

As razões relatadas para manutenção do comportamento foram preocupação com prevenção ( 23 de 31 participantes), percepções de aumento de força e melhor controle de movimentos dos braços (20 de 31 participantes) e melhora no desempenho de atividades de fisioterapia em aulas práticas (8 de 31 participantes). As razões apresentadas são diferentes das normalmente citadas em programas de exercícios 
gerais, como bem estar e melhora da saúde ${ }^{13}$, e acreditamos que isto se deva exclusivamente às características do programa avaliado e sua população.

As razões relatadas em relação ao abandono foram falta de tempo (15 dos 17 estudantes) e opção por outras prioridades ( 7 dos 17 participantes). $\mathrm{O}$ fator falta de tempo geralmente é o mais citado como fator relacionado ao abandono em estudos que envolvem exercícios físicos e, não foi diferente neste estudo.

A manutenção implicou em melhora adicional de força para o subgrupo manutenção $(\mathrm{n}=31)$ de $14 \%$, em média, sendo esta alteração estatisticamente significativa $(p=0,007)$, e o abandono causou uma perda média de $19 \%$ para o subgrupo abandono $(\mathrm{n}=17)$, sendo esta perda estatisticamente significante $(\mathrm{p}=0,02)$, mas não o suficiente para que estes sujeitos voltassem aos valores coletados em $\mathrm{T} 1$, antes do treinamento. A força muscular se mostrou um indicador adequado para o estudo de manutenção, podendo inclusive, em estudos futuros ou na prática clínica, serem utilizados como reforçadores ${ }^{14}$.

Dos 48 participantes que completaram a pesquisa, todos reproduziram os exercícios treinados de forma completa, mas somente 32 executaram os exercícios de forma correta, isto é, controlando as possíveis compensações musculares típicas de cada exercício. Os dezesseis sujeitos que não conseguiram realizar adequadamente os exercícios pertenciam ao subgrupo abandono. Associamos estes achados à elaboração cuidadosa do programa que deu grande valor aos aspectos didáticos do ensino de exercícios físicos ${ }^{15} \mathrm{e} o$ fato de que a prática pela manutenção manteve a memória correta dos cuidados a serem realizados durante a prática dos exercícios.

A comparação da manutenção entre homens e mulheres mostrou que não houve diferença entre os grupos, nem em número (três abandonos masculino e 14 abandonos femininos, compatível com a população inicial), nem nos efeitos do treinamento e da manutenção.

\section{CONCLUSÃO}

O programa proposto produziu melhora significativa na força muscular. A manutenção se deu em índices superiores aos encontrados na literatura. Os comportamentos envolvidos com manutenção se relacionaram com as discussões teóricas propostas no programa.

A forma de ensino e treinamento foi eficiente para o aprendizado, a memorização foi dependente da manutenção, produzindo alto índice de reprodutibilidade correta destes seis meses após o final do programa.

A manutenção da prática de exercícios implicou em melhora adicional de força muscular.

Acreditamos que este tipo de programa deva ser oferecido como rotina para alunos de cursos de Fisioterapia e que haja acompanhamento dos alunos por um período de tempo maior, quando da certeza da mudança de comportamento definitivamente instalada.

CAROMANO, F. A.; CARLIS, M.; PANADES, L. dos S. C.; IDE, M. R.; ASSIS, S. M. B. de. Extremity superior exercises practice among Physiotherapy students - six-month follow-up study. Rev. Ter. Ocup. Univ. São Paulo, v.19, n. 1, p. 56-60, jan./abr. 2008.

\begin{abstract}
Objective: To assess the maintenance and effects of a six-month extremity superior exercises practice among Physiotherapy students. Setting: This study was conduced from 1999 to 2003, with five groups of 10 students of Physiotherapy Course of Physiotherapy, Medicine School, University of São Paulo. Participants: Fifty students, 10 male and 40 female (mean age 20,4 \pm 2,1 years) who was engaged in Therapeutic Manual Resources discipline. Procedure: Program consisted in a sequence of 20 exercises that had for objective the gain of muscular force, trained using functional patterns of movements. The exercises were trained during ten sessions of one hour, using 20 minutes for discussion about studies showing occupational muscleskeletical lesions in professional physiotherapists and 40 minutes for training. Measures and behavioral evaluation: Eight isometric muscular force tests of flexion and extension of superior extremity were measured using digital dinamometry (Laffayetti Instruments) An open question and the demonstration of the trained sequence of exercises were used to evaluated the behavior. Data analysis: Study on the effect of exercises used the average of the eight muscular force tests, considering the right superior member, obtained in T1 and compared with T2, using paired Student's $t$ Test. The same analysis was conduced comparing T2 and T3, resulting in a indicative of maintenance. The differences found between T1, T2 and T3 were also transformed in percentage. We also compared male and female subgroups. The answers to the open question had a quantitative analysis and collected information were grouping by theme. Results: Forty eight of the original 50 subjects completed the six-month
\end{abstract}


follow-up evaluation. The program produced a statistical significant improvement in muscular force $(\mathrm{p}=0,0067)$ with increase of $32 \%$. Thirty one subjects reported continued in some form of the practice of exercises and others 17 related no practice during the preceding six-month. Reasons to maintenance involved intention of prevention and better control of superior extremity movements. Reasons to abandonment included no time and others priorities. Gender was not significant predictor of exercise behavior. Conclusions: The program produced significant improvement in the muscular force. The maintenance reached superior indexes to that found in the literature. The behaviors involved with maintenance linked with the theoretical discussions proposed in the program. The didacticism used in training was efficient for exercises memorization, producing high index of correct reproducibility six months after the end of the program. The maintenance of the practice of exercises implied in additional improvement of muscular force. We believe that this kind of activity should be offer habitually for Physiotherapy students.

KEY WORDS: Preventive maintenance. Exercise therapy/manpower. Exercise. Students, health occupations. Muscle strength.

\section{REFERÊNCIAS}

1. MARTIN, J. E.; DUBBERT, P. M. Exercise applications and promotion in behavioral medicine: current status and future directions. Health Psychology, v. 50, p. 1004-1017, 1982.

2. HERBERT, L.; TEAGUE, M. D. Exercise adherence and older adults. Activities. Adaptation Aging, v. 13, p. 91-105, 1989.

3. FRAZER, S.; SPINK, K. Examining the role of social support and group cohesion in exercise compliance. J. Behav. Med., v. 25, p. 233-49, 2002.

4. BODGAN, R. C.; BIKEEN, S. K. Qualitative research for education: na introduction to theary and methods. Boston: Allyn. Bacon, 1992.

5. BRUS, H.; VAN de LAAR, M.; TAAL, E.; RASKER, J.; WIEGMAN, O. Compliance in rheumatatoid arthritis and the role of formal patient education. Semin. Arthritis Rheum., v. 26, p. 702-710, 1997.

6. YLINEM, J.; TAKAFA, E. P.; NYKÄNEN, M. Active neck muscle training in the treatment of chronic neck pain in women. JAMA, n. 289, p. 2509-16, 2003.

7. DORTCH, H. L.; TROMBLY, C. A. The effect of education on hand use with industrial workers in repetitive jobs. Am. J. Occup. Ther, 44: 777-82, 1990.

8. BORK, B. E.; COOK, T. M.; ROSECRANE, J. C. Work-related muscleskeletal disorders among physical therapists. Phis. Ther., n. 76 , p. 827-835, 1996.

9. HAYES, K. Behaviors that cause clinical instructors question the clinical competence of physical therapists students. Phys. Ther., n. 79, p. 653-667, 1999.

10. HOLDER, N. L.; CLAARK, H. A.; DIBLASIO, J. M. Cause prevalence and response to occupational muscleskeletal injuries reported by physical therapists. Phys. Ther., n. 79, p. 642-652, 1999.

11. BAHANNON, R. W.; ANDREWS, A. W. Interrater reability of hand-held dynamometry. Phys. Ther., n. 67, 931-933, 1987.

12. BAHANNON, R. W.; SAUNDERS, N. Hand-held dynamometry: a single trial may be adequate for measuring muscle strength in health individuals. Physiother. Canada, v. 42, n. 1, p. 6-9, 1990.

13. DISHMAN, R. K. Exercise adherence. In: SINGER, R.N., MURPHY M., TENNANT L. K. (eds.). Handbook of Research in Sport Psychology. New York: McMillan. 1993. Cap. 36.

14. KIRKCALDY, B. D.; FURNHAM, A.; SHEPHARD, R. Attitudes towards health and illness among exercisers and nonexercisers. Stress Med., v.10, n. 1, p. 21-26, 1994.

15. MARCUS, B. H. Exercise behavior and strategies for intervention. Res. Quarterly Exercise Sport, v. 66, n. 4, p. 319-323, 1995.

16. MARCUS, B. H.; RAKOWSKI, W.; ROSSI, J. S. Assessing motivational readyness and decision making for exercise. Health Psychol., v. 11, n. 4, p. 257-261, 1992.

17. TURATO, E. R. Tratado de metodologia de pesquisa clínico quantitativa. Rio de Janeiro: Vozes, 2003. p. 685. 